\title{
Fatores de risco modificáveis para fasciíte plantar em pacientes não atletas e proposta de um novo sistema de avaliação objetiva - RKISP
}

\section{Modifiable Risk Factors of Plantar Fasciitis in Non- Athletic Patients and Proposal of a New Objective Assessment System - RKISP}

\author{
Ranjeet Choudhary ${ }^{10}$ Kishor Kunal $\left.{ }^{2(}\right)$ \\ 1 Professor Assistente, AlIMS, Raipur, Chattisgarh, India \\ 2 Department of Orthopedics, AlIMS, Jodhpur, Rajasthan, India \\ Endereço para correspondência Kishor Kunal, Departamento de \\ Ortopedia, AlIMS, Jodhpur, Rajasthan, 342005, India \\ (e-mail: drkunal2408@gmail.com).
} Rev Bras Ortop 2021;56(3):368-371.

\section{Resumo}

\section{Palavras-chave}

- fatores de risco

- fasciíte plantar

- dor crônica

\section{Abstract}

recebido

17 de Fevereiro de 2020

aceito

06 de Julho de 2020

Publicado on-line

Outubro 29, 2020
Objetivo Determinar os fatores de risco modificáveis associados à gravidade da fasciíte plantar e formular um sistema objetivo de pontuação para indexação da doença na população não atlética.

Métodos Estudo observacional prospectivo. A principal medida de desfecho foi a associação de um fator de risco modificável, mensurada pelo valor de $\mathrm{R}$ (coeficiente de Pearson) e pelo nível de significância de $p<0,05$.

Resultados Em uma amostra de 50 pacientes, o índice de massa corporal (IMC) e calçados com amortecimento inadequado foram associados de maneira significativa à dor na fasciíte plantar. Todos os demais fatores de risco eram não modificáveis ou não apresentaram associação significativa.

Conclusão Com base nos dados à disposição e sua interpretação, um índice, denominado Índice de Ranjeet-Kunal de Pontuação da Fasciíte Plantar (RKISP, em inglês) , foi formulado e utilizado com sucesso não apenas na classificação da fasciíte plantar, mas também na determinação do prognóstico de seu tratamento conservativo, auxiliando a escolha da modalidade terapêutica.

Objective To determine the modifiable risk factors associated with severity of plantar fasciitis and to formulate an objective scoring system for indexing plantar fasciitis in the non-athletic population.

Methods This was a prospective observational study. The main outcome measure was association of a modifiable risk factor, which was kept by means of the
DOI https://doi.org/ $10.1055 / \mathrm{s}-0040-1716762$. ISSN $0102-3616$.
(C) 2020. Sociedade Brasileira de Ortopedia e Traumatologia. All rights reserved.

This is an open access article published by Thieme under the terms of the Creative Commons Attribution-NonDerivative-NonCommercial-License, permitting copying and reproduction so long as the original work is given appropriate credit. Contents may not be used for commercial purposes, or adapted, remixed, transformed or built upon. (https://creativecommons.org/ licenses/by-nc-nd/4.0/)

Thieme Revinter Publicações Ltda., Rua do Matoso 170, Rio de Janeiro, RJ, CEP 20270-135, Brazil 
Pearson coefficient (R-value) and the level of significance, which was determined as $p<0.05$.

Result In a sample size of 50 patients, the body mass index (BMI) and ill-cushioned shoes were found to be significantly associated with pain in plantar fasciitis. All the other risk factors were either non-modifiable or had no significant association.

Keywords

- risk factors

- plantar fasciitis

- chronic pain
Conclusion Based on available data and further interpretation, an index was be formulated, named as Ranjeet-Kunal Index for Scoring Plantar fasciitis (RKISP), which can be successfully used for not only grading plantar fasciitis but also prognosticating the conservative management of the same, thus deciding the modality of treatment.

\section{Introdução}

A fasciíte plantar (FP) é a causa mais comum de dor crônica no calcanhar plantar. ${ }^{1}$ É uma síndrome degenerativa da fáscia plantar resultante de traumas repetidos em sua origem no calcâneo. A flexibilidade do pé, a tíbia vara, o tornozelo em equino, o retropé varo, o antepé varo, o antepé valgo compensado e a desigualdade no comprimento dos membros podem alterar a força normal de pronação. A maior pronação aumenta a tensão na banda central anatômica da fáscia plantar e pode causar fasciíte plantar. ${ }^{2}$ A corrida também foi considerada um fator de risco para a fasciíte plantar. $^{3}$ Ganho de peso, atividade laboral, variações anatômicas, problemas biomecânicos, esforço excessivo e calçados inadequados são fatores que contribuem para o desenvolvimento da doença. ${ }^{4}$

$O$ índice de massa corporal (IMC) elevado (especialmente a contribuição do peso) tem forte associação com a FP na população não atlética. ${ }^{5,6}$ Os fatores de risco podem ser divididos em intrínsecos e extrínsecos. Os fatores intrínsecos podem ser subdivididos em anatômicos e biomecânicos, enquanto os fatores extrínsecos incluem mau alinhamento, caminhar em superfície dura, andar descalço, suporte prolongado de peso e uso de calçados de má qualidade. Os fatores anatômicos são obesidade, pé chato, pé cavo e encurtamento do tendão calcâneo; os fatores biomecânicos são pronação excessiva, flexão dorsal limitada do tornozelo e fraqueza dos músculos intrínsecos e dos músculos flexores plantares. $^{5,7}$

Não há um sistema específico de pontuação para a gravidade da fasciíte plantar e avaliação de seu tratamento. Embora outras pontuações comuns, como a escala visual analógica (EVA), o Foot and Ankle Ability Measure (FAAM), o Foot and Ankle Disability Index (FADI) e o American Foot and Ankle Society (AOFAS), sejam tradicionalmente utilizadas para avaliações objetivas e subjetivas da fasciíte plantar, elas são generalistas demais para os pés e tornozelos (FAAM, FADI e AOFAS) ou se referem à dor em qualquer outra parte do corpo (EVA). ${ }^{8-11}$

Neste estudo, buscamos descobrir fatores de risco modificáveis que poderiam ser empregados para a avaliação da fasciíte plantar e usá-los para formulação de um sistema de pontuação para quantificação da doença à apresentação e durante o acompanhamento.

\section{Metodologia (-Figura 1)}

Em um estudo realizado na unidade de saúde terciária LHMC, Nova Délhi, Índia, denominado “Avaliação de várias causas de dor no calcanhar e eficácia da injeção autóloga de plasma rico em plaquetas em casos de fasciíte proximal com insucesso do tratamento conservativo", os fatores de risco modificáveis foram analisados com a ajuda do coeficiente de Pearson (R).

O principal achado da fasciíte plantar é a dor crônica no calcanhar e foi feita uma tentativa de encontrar uma associação entre a EVA e os fatores de risco. O IMC, o ajuste dos calçados e seu amortecimento e o nível de atividade são variáveis que, segundo a literatura, podem afetar a dor na fasciíte plantar. Idade e sexo foram considerados fatores de risco não modificáveis, assim como todos os outros fatores anatômicos que requerem correção cirúrgica. Com base na observação de associação significativa, um índice foi formulado para avaliação objetiva da fasciíte plantar à apresentação e após o tratamento. Todos os fatores de risco que necessitam de intervenção cirúrgica para modificação foram considerados não modificáveis devido a razões técnicas. O IMC (valores correspondentes à obesidade I e II, isto é, > 24,99 e > 29,99, segundo a Organização Mundial da Saúde) e a EVA são variáveis quantitativas, e as demais são variáveis qualitativas; ${ }^{12,13}$ portanto, a quantificação foi feita com base na literatura para classificação no sistema de pontuação. De acordo com a significância das associações já mencionadas com a sintomatologia, propomos um novo sistema de indexação para avaliação quantitativa objetiva da fasciíte plantar. Criamos um sistema de avaliação imparcial para quantificação da doença à apresentação e durante seu tratamento, que não é passível de avaliação subjetiva, que tende a quantificar o "problema" associado à doença, e não a doença em si e pode apresentar alta variação interobservador e intraobservador.

\section{Resultados}

No total, 50 pacientes, 23 homens e 27 mulheres, com idade média de 41,94 anos e desvio-padrão de 8,94 , foram estudados. O IMC médio foi de $28,79 \mathrm{~kg} / \mathrm{m}^{2}$ (29,82 em mulheres e 27,57 em homens). A correlação de Pearson de vários fatores com a EVA é mostrada na - Tabela 1.

O IMC apresentou forte correlação positiva com a EVA, com valor de $\mathrm{R}$ de 0,64 e valor de $p<0,0001$. Para calçados bem 
Tabela 1 Valor de R (coeficiente de Pearson) e nível de significância da associação entre gravidade da doença (dor) e fatores de risco modificáveis

\begin{tabular}{|l|l|l|}
\hline & Valor de $\mathbf{R}$ & Valor de $\boldsymbol{p}$ \\
\hline Índice de massa corporal & 0,64 & $<0,0001$ \\
\hline Calçados bem ajustados & $-0,16$ & 0,26 \\
\hline Amortecimento íntegro & $-0,41$ & 0,0033 \\
\hline Nível de atividade & 0,05 & 0,72 \\
\hline
\end{tabular}

ajustados apresentaram correlação negativa, com valor de R de - 0,16 e valor de $p$ de 0,26 , sem associação significativa. Calçados com bom amortecimento apresentaram forte correlação negativa, com valor de $\mathrm{R}$ de - 0,41, e associação significativa com os sintomas do paciente, com valor de $p$ igual a 0,0033 . A atividade foi dividida em três níveis (sedentarismo, atividade moderada e atividade intensa) e, embora tivesse uma pequena correlação positiva, com valor de $\mathrm{R}$ de 0,05 , não foi significativamente associada à sintomatologia $(p=0,72)$

De acordo com os níveis de significância, um novo sistema de indexação para avaliação quantitativa da fasciíte plantar foi proposto com base na EVA, no IMC de obesidade I e obesidade II e no amortecimento dos calçados (veja detalhes em Discussão).

\section{Discussão}

A fasciíte plantar é uma causa de dor crônica no calcanhar. A cronicidade faz com que o indivíduo se acomode à dor e às apresentações tardias habituais, mas os médicos muitas vezes deixam de entender que não apenas a doença é crônica e que os fatores de risco também podem ser crônicos - assim, por um lado, podem não causar sofrimento significativo para o paciente, mas podem contribuir de forma significativa para a progressão da doença. Podemos entender isso em termos dos fatores de risco mencionados, como obesidade e calçados com amortecimento inadequado. O calçado utilizado por uma pessoa é mais uma declaração de seu conforto do que de seu status e, portanto, calçados com bom amortecimento podem parecer convidativos, mas os indivíduos tendem a se acomodar a seus sapatos com o passar do tempo - o que age como um veneno lento e potente para a fáscia plantar. Da mesma forma, a aquisição da obesidade é geralmente demorada e o paciente se acostuma até ter graves problemas de saúde, que não são observados em pacientes jovens com obesidade de grau I. Assim, pode-se entender que, embora a cronicidade em si seja uma manifestação da fasciíte plantar, ela indica fatores de risco negligenciados, como calçados com amortecimento inadequado (que, em muitos casos, podem ser bastante confortáveis para o paciente) e obesidade especialmente se recente e em fases iniciais - quando têm menos impactos à saúde. Também deve ser entendido que esse sistema de pontuação é para a população não atlética, que não apresenta desgaste da fáscia plantar.

Agora, vamos a cada um dos componentes do Índice de Ranjeet-Kunal para Pontuação da Fasciíte Plantar(RKISP). Com
Tabela 2 Índice de Ranjeet-Kunal para Pontuação da Fasciíte Plantar (RKISP) (Para ser avaliado pelo RKISP, o paciente precisa apresentar dor crônica no calcanhar com pontuação > 4,5 na escala visual analógica [EVA]. Veja mais detalhes no texto)

\begin{tabular}{|l|l|l|}
\hline Índice de massa corporal & $>24,99$ (obesidade I) & 1 \\
\hline & $>29,99$ (obesidade II) & 2 \\
\hline Amortecimento desgastado & & 1 \\
\hline EVA & $>4,5$ & 1 \\
\hline & $>7,5$ & 2 \\
\hline Total & 5 \\
\hline
\end{tabular}

Abreviatura: EVA, escala visual analógica.

base nos dados já mencionados, pode-se entender que o IMC, como indicado em estudos anteriores, tem uma forte correlação positiva com a sintomatologia e a EVA na fasciíte plantar. Como a obesidade tem associação muito significativa, pode ser subdividida de acordo com a classificação da OMS da população indiana, em que o IMC $>24,99$ indica obesidade I e o IMC > 29,99 indica obesidade II. $^{12}$ Essa classificação é para a população asiática e pode precisar ser alterada para a população ocidental, pois depende de seu cenário; além disso, pode ser modificada conforme a classificação da OMS de obesidade na população ocidental. Para o índice, 1 ponto é atribuído à obesidade I e 2 pontos à obesidade II.

A pontuação de EVA é comumente usada para quantificação da dor em pacientes com diferentes patologias. $\mathrm{O}$ EVA $>7,5$ é uma medida de dor intensa sofrida pelo paciente. ${ }^{13}$ A EVA $<4,5$ define a dor comum e, portanto, não foi incluída no índice. Dois pontos são atribuídos à dor intensa, com EVA $>7,5$.

Como calçados com bom amortecimento apresentaram forte correlação negativa com a sintomatologia, torna-se óbvio que a troca de um sapato com amortecimento de má qualidade tem efeito dramático tanto na prevenção quanto no tratamento da sintomatologia da fasciíte plantar. Por isso, é parte importante da avaliação quantitativa da doença.

A - Tabela 2 mostra os três parâmetros considerados na avaliação objetiva da fasciíte plantar antes e depois do tratamento. O RKISP inclui esses três parâmetros, com soma máxima de 5 e valor mínimo de 1 (o critério mínimo para uso do índice foi a pontuação de EVA $>4,5$, ou seja, os critérios de avaliação de dor descritos por Hawker et al. ${ }^{13}$ ). $\mathrm{O}$ RKISP pode ser usado na classificação da fasciíte plantar.

\section{Implicações do Índice de Ranjeet-Kunal para Pontuação da Fasciíte Plantar}

- Avaliação objetiva à apresentação da fasciíte plantar e subsequente acompanhamento. A avaliação objetiva não apenas auxilia a quantificação imparcial da doença, mas também uniformiza os protocolos de tratamento e pesquisa.

- Classificação da fasciíte plantar. A classificação pode ser baseada em outros parâmetros que podem piorar a dor em pacientes com fasciíte plantar. 


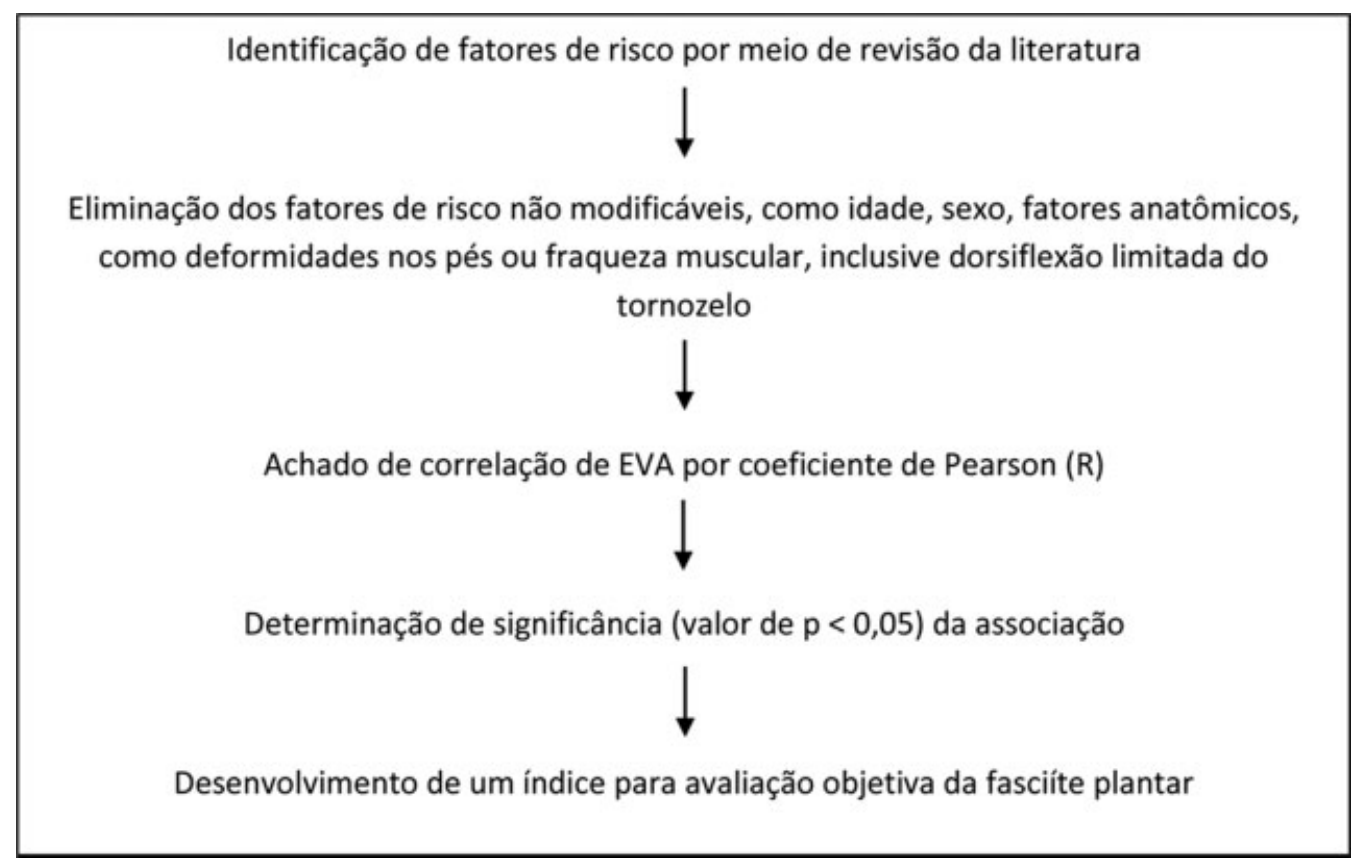

Fig. 1 Fluxograma da metodologia empregada para determinação do sistema objetivo de pontuação para avaliação de fascíte plantar em pacientes não atletas. EVA, escala visual analógica.

- O prognóstico do tratamento conservativo é inversamente proporcional ao índice. Assim, um índice maior indica a maior possibilidade de tratamento conservativo. Por exemplo, um paciente com índice 2 e EVA de 9/10 que usa sapato com bom amortecimento e apresenta IMC $<24,99$ apresenta prognóstico inferior a um paciente com índice 4, calçados desgastados, obesidade de grau II e EVA igual a 5 sob tratamento conservativo.

\section{Conclusão}

O RKISP pode fazer uma avaliação objetiva, quantificando a doença em termos de problemas e fatores de risco. Este índice também pode ser utilizado para a classificação e determinação do prognóstico da fasciíte plantar à apresentação e durante o tratamento, como já discutido. O RKISP precisa ser validado em pesquisas com amostras maiores. Os autores também recomendam o uso de sistemas de avaliação objetiva para fasciíte plantar, além dos sistemas subjetivos amplamente utilizados.

Conflito de Interesses

Os autores declaram não haver conflitos de interesses.

\section{Referências}

1 Irving DB, Cook JL, Menz HB. Factors associated with chronic plantar heel pain: a systematic review. J Sci Med Sport 2006;9(12):11-22, discussion 23-24

2 Cornwall MW, McPoil TG. Plantar fasciitis: etiology and treatment. J Orthop Sports Phys Ther 1999;29(12):756-760

3 Lopes AD, Hespanhol Júnior LC, Yeung SS, Costa LO. What are the main running-related musculoskeletal injuries? A Systematic Review. Sports Med 2012;42(10):891-905
4 Roxas M. Plantar fasciitis: diagnosis and therapeutic considerations. Altern Med Rev 2005;10(02):83-93

5 Tahririan MA, Motififard M, Tahmasebi MN, Siavashi B. Plantar fasciitis. J Res Med Sci 2012;17(08):799-804

6 van Leeuwen KD, Rogers J, Winzenberg T, van Middelkoop M. Higher body mass index is associated with plantar fasciopathy/ 'plantar fasciitis': systematic review and meta-analysis of various clinical and imaging risk factors. Br J Sports Med 2016;50(16): 972-981

7 Schwartz EN, Su J. Plantar fasciitis: a concise review. Perm J 2014; 18(01):e105-e107

8 Choudhary R, Chaudhary P, Sud A, Ramavat S. Efficacy Of Autologous PlateletRich Plasma Injection In Cases Of Proximal Plantar Fascitis Which Have Failed Conservative Management. Int J Sci Res (Ahmedabad) 2017;6(08):372-374

9 Verma D, Kumar S, Dhammi IK, Kumar R, Kapoor R, Mittal A. Role of platelet-rich plasma versus corticosteroid injection in the treatment of plantar fasciitis: A comparative study. J Orthop Dis Traumatol 2019;2(02):26-30

10 Reddy RM, Kumar AV, Shaik T, Rudra S. The role of PRP injection in the management of plantar fasciitis: The analytical prospective study in 60 patients. Nat J Clin Orthop 2019;3(03):100-104

11 Akl RA, El Sadek M, El Dayem AA, Mostafa MS. Platelet Rich Plasma versus Corticosteroid Local Injection Results in Treatment of Planter Fasciitis in New-Cairo. Zagazig Univ Med J 2019; 25:665-672

12 World Health Organization. The Asia-Pacific perspective: redefining obesity and its treatment. Sydney: Health Communications Australia; 2000

13 Hawker GA, Mian S, Kendzerska T, French M. Measures of adult pain: Visual analog scale for pain (vas pain), numeric rating scale for pain (nrs pain), McGill pain questionnaire (mpq), short-form mcgill pain questionnaire (sf-mpq), chronic pain grade scale (cpgs), short form-36 bodily pain scale (sf-36 bps), and measure of intermittent and constant osteoarthritis pain (icoap). Arthritis Care Res (Hoboken) 2011;63(Suppl 11):S240-S252 